\title{
Solar Neutrino Cross Sections and Nuclear Beta Decay*
}

\author{
JoHN N. BaHCALI \\ California Institute of Technology, Pasadena, California
}

(Received 10 February 1964)

\begin{abstract}
The neutrino-producing reactions that are believed to occur in the interior of the sun are reviewed and the predicted neutrino fluxes from these reactions are presented. The relevant absorption cross sections for neutrino-induced transitions from the ground state of $\mathrm{Cl}^{37}$ to various states in $\mathrm{Ar}^{37}$ are then calculated. The nuclear model adopted in these calculations is also used to predict the decay rates and branching ratios for a number of nuclei with $1 d_{3 / 2}{ }^{n}$ configurations; comparisons with experiment are made wherever possible and additional tests are suggested. A method for extracting Gamow-Teller matrix elements from mixed transitions between members of the same isotopic multiplet is then illustrated. The theory of bound-state neutrino capture is also outlined and applied to the proposed solar neutrino experiment. In an Appendix, some neutrino cross sections that are useful in interpreting the reactor experiment of Davis are presented.
\end{abstract}

\section{INTRODUCTION}

$T$ HE theory of nuclear energy generation in the sun can be tested directly ${ }^{1,2}$ by observing the terrestrial rate of the reaction $\mathrm{Cl}^{37}\left(\nu_{\text {solar }}, e^{-}\right) \mathrm{Ar}^{37}$. In this paper, we present some of the theoretical considerations that underlie the proposed ${ }^{2}$ solar neutrino experiment and suggest a number of laboratory experiments that could reduce the uncertainties in the interpretation of Davis's solar neutrino experiment.

In Sec. II, the neutrino-producing reactions that are believed to occur in the interior of the sun are reviewed; the predicted fluxes from these reactions are given in Sec. III. The relevant absorption cross sections for transitions between the ground states of $\mathrm{Cl}^{37}$ and $\mathrm{Ar}^{37}$ are presented in Sec. IV. In Sec. V, the theory of boundstate neutrino capture is outlined and applied to the proposed solar neutrino experiment. In Sec. VI, the cross sections for neutrino-induced transitions from the ground state of $\mathrm{Cl}^{37}$ to various excited states in $\mathrm{Ar}^{37}$ are calculated. The nuclear model adopted in Sec. VI is then used to predict, in Sec. VII, the decay rates and branching ratios for a number of other nuclei with $1 d_{3 / 2}{ }^{n}$ configurations; comparisons with experiment are made wherever possible and additional tests are suggested. The total predicted number of solar neutrino captures per terrestrial $\mathrm{Cl}^{37}$ atom per sec is given in Sec. VIII. In an Appendix, some cross sections that are useful in interpreting the reactor experiment of Davis ${ }^{3}$ are presented.

\section{NEUTRINO-PRODUCING REACTIONS}

The following reactions that are believed to occur in the interior of the sun produce neutrinos with continuous energy spectra ${ }^{4,5}$ :

${ }^{*}$ Supported in part by the U. S. Office of Naval Research and in part by the National Aeronautics and Space Administration.

${ }^{1}$ J. N. Bahcall, Phys. Rev. Letters 12, 300 (1964).

2 R. Davis, Jr., Phys. Rev. Letters 12, 302 (1964).

${ }^{3}$ R. Davis, Jr., Phys. Rev. 97, 766 (1955); in Proceedings of the First UNESCO International Conference, Paris, 1957 (Pergamon Press, London, 1958), Vol. 1.

${ }^{4}$ W. A. Fowler, Mem. Soc. Roy. Sci. Liege 3, 207 (1960)

${ }^{5}$ P. D. Parker, J. N. Bahcall, and W. A. Fowler, Astrophys. J. 139,602 (1964). This article contains a discussion of the uncertain-

$$
\begin{aligned}
& \mathrm{H}^{1}+\mathrm{H}^{1} \rightarrow \mathrm{H}^{2}+e^{+}+\nu \quad(0.420 \mathrm{MeV}), \\
& \mathrm{B}^{8} \rightarrow \mathrm{Be}^{8 *}+e^{+}+\nu \quad(14.06 \mathrm{MeV}), \\
& \mathrm{N}^{13} \rightarrow \mathrm{C}^{13}+e^{+}+\nu \quad(1.20 \mathrm{MeV}), \\
& \mathrm{O}^{15} \rightarrow \mathrm{N}^{15}+e^{+}+\nu \quad(1.74 \mathrm{MeV}) .
\end{aligned}
$$

The numbers given in parenthesis are, except for reaction (2), the maximum neutrino energies for the respective reactions. The state in $\mathrm{Be}^{8}$ that is populated by the beta decay of $\mathrm{B}^{8}$ is approximately $2 \mathrm{MeV}$ wide; hence, the number given in parenthesis beside reaction (2) is the maximum neutrino energy corresponding to $\mathrm{B}^{8}$ beta decay to the peak of the first $2^{+}$resonance in $\mathrm{Be}^{8}$. If $\mathrm{Li}^{4}$ were only slightly heavier than $\mathrm{He}^{3}$ and a proton, the following decay would also be important:

$$
\mathrm{Li}^{4} \rightarrow \mathrm{He}^{4}+e^{+}+\nu \quad(\sim 18.8 \mathrm{MeV})
$$

However, arguments based upon the preliminary experiment of Davis ${ }^{2}$ and the cross sections presented in Secs. $\mathrm{V}$ and VI have already been used to show ${ }^{1,5}$ that $\mathrm{Li}^{4}$ is heavier than $\mathrm{He}^{3}$ plus a proton by more than $20 \mathrm{keV}$ and that $\mathrm{Li}^{4}$ does not play a significant role in the hydrogen-burning reactions in the sun.

The following reactions produce neutrinos with discrete energies, except for a small thermal broadening due to the spread in intitial electron energies and, in the case of $\mathrm{B}^{8}$, a $2 \mathrm{MeV}$ spread due to the width of the particle-unstable final state of $\mathrm{Be}^{8}$ :

$$
\begin{aligned}
\mathrm{H}^{1}+\mathrm{H}^{1}+e^{-} \rightarrow \mathrm{H}^{2}+\nu & (1.44 \mathrm{MeV}), \\
\mathrm{He}^{3}+e^{-} \rightarrow \mathrm{H}^{3}+\nu & (-0.018 \mathrm{MeV}), \\
\mathrm{Be}^{7}+e^{-} \rightarrow \mathrm{Li}^{7}+\nu & (0.861 \mathrm{MeV}, 90 \% ; \\
& 0.383 \mathrm{MeV}, 10 \%), \\
\mathrm{B}^{8}+e^{-} \rightarrow \mathrm{Be}^{8}+\nu & (15.08 \mathrm{MeV}), \\
\mathrm{N}^{13}+e^{-} \rightarrow \mathrm{C}^{13}+\nu & (2.22 \mathrm{MeV}), \\
\mathrm{O}^{15}+e^{-} \rightarrow \mathrm{N}^{15}+\nu & (2.76 \mathrm{MeV}) .
\end{aligned}
$$

ties in the nuclear parameters for the most important neutrinoproducing reactions. 
TABLE I. Neutrino fluxes from reactions (1)-(4) and (8)..$^{\mathrm{a}}$

\begin{tabular}{cc}
\hline Reaction & $\begin{array}{c}\text { Neutrino flux at earth } \\
\left.\text { (Nos. } \mathrm{cm}^{-2} \mathrm{sec}^{-1}\right)\end{array}$ \\
\hline 1 & $(5.3 \pm 0.6) \times 10^{+10}$ \\
2 & $(2.5 \pm 1) \times 10^{+7}$ \\
3 & $(1 \pm 0.5) \times 10^{+9}$ \\
4 & $(1 \pm 0.5) \times 10^{+9}$ \\
8 & $(1.2 \pm 0.5) \times 10^{+10}$ \\
\hline
\end{tabular}

a Adapted from the work of Sears, Ref. 7.

\section{NEUTRINO FLUXES}

\section{A. Reactions (1)-(4) and (8)}

The neutrino fluxes from the sun due to reactions (1)-(4) and (8) have been calculated by Sears and others ${ }^{6-8}$ using detailed models of the sun. Sears ${ }^{7}$ has also investigated the uncertainties in the predicted fluxes due to uncertainties in nuclear cross sections, as well as solar composition, opacity, and age. The estimated neutrino fluxes, derived from the work of Sears, from reactions (1)-(4) and (8) are given in Table I. The choice of "best" values for the fluxes presented in Table I is somewhat arbitrary since the predicted fluxes depend upon nuclear and solar parameters that are imperfectly known. The reader is referred to the original work ${ }^{7}$ for a clear discussion of the sources of uncertainties.

Note in Table I that the neutrinos from reaction (1) ( $p-p$ neutrinos) are the most numerous and the neutrinos from reaction (2) ( $\mathrm{B}^{8}$ neutrinos) are the least numerous.

\section{B. $\mathrm{H}^{1}+\mathrm{H}^{1}+e^{-} \rightarrow \mathrm{H}^{2}+v$}

The relative probability of reactions (6) and (1) is approximately equal to ${ }^{8,9}$ the final-state phase space for reaction (6) divided by the final-state phase space for reaction (1). The phase-space factor for reaction (6) has been calculated by Bahcall, ${ }^{10}$ and the phasespace factor for reaction (1) has been calculated by Salpeter. ${ }^{11}$ Inserting the parameters of the basic model of Sears $^{7}$ in the above-cited phase-space factors, we find that reaction (6) is responsible for only $0.3 \%$ of the proton-proton reactions at the center of the sun. The solar average (computed by a hand integration with the basic model of $\operatorname{Sears}^{7}$ ) of the relative probability for reactions (6) and (1) is about 0.002 . Thus, the neutrino flux at the earth's surface from reaction (6) is approximately $1 \times 10^{+8}$ neutrinos per $\mathrm{cm}^{2}$ per sec. It

${ }^{6}$ J. N. Bahcall, W. A. Fowler, I. Iben, Jr., and R. L. Sears, Astrophys. J. 137, 344 (1963). See also R. L. Sears, Mem. Soc. Roy. Sci. Liege 3, 479 (1960).

${ }^{7}$ R. L. Sears, Astrophys. J. (to be published).

${ }^{8}$ See also the article by $\mathrm{H}$. Reeves and P. Pochoda (to be published) for similar predictions based upon a somewhat different model for the sun.

${ }^{9}$ E. Schatzman, White Dwarfs (North-Holland Publishing Company, Amsterdam, 1958).

${ }_{10}$ J. N. Bahcall, Phys. Rev. 126, 1143 (1962).

11 E. E. Salpeter, Phys. Rev. 88, 547 (1952). is shown in Sec. IV that this neutrino flux, when combined with the appropriate neutrino absorption cross section, is too small to be of importance in Davis's proposed experiment.

$$
\text { C. } \mathrm{He}^{3}+e^{-} \rightarrow \mathrm{H}^{3}+v
$$

Pochoda and Reeves ${ }^{8}$ have pointed out that endoergic reaction (7) gives rise to both neutrinos and antineutrinos. The relevant sequence of decays is

and

$$
\mathrm{He}^{3}+e^{-} \rightarrow \mathrm{H}^{3}+\nu
$$

$$
\mathrm{H}^{3} \rightarrow \mathrm{He}^{3}+e^{-}+\bar{\nu} .
$$

Bahcall and $\mathrm{Wolf}^{12}$ have recently calculated the relative probability for the $\mathrm{He}^{3}\left(e^{-}, \nu\right) \mathrm{H}^{3}$ and the $\mathrm{He}^{3}\left(\mathrm{He}^{3}, 2 p\right) \mathrm{He}^{4}$ reactions. They find, for typical solar conditions, that reaction (7) occurs only about two times for every $10^{+5}$ proton-proton completions. Moreover, the $\mathrm{H}^{3}(p, \gamma) \mathrm{He}^{4}$ reaction occurs about $10^{+9}$ times faster in the sun's interior than the beta decay of tritium, reaction $\left(7^{\prime}\right)$. Hence, the flux of low-energy neutrinos and antineutrinos from reactions $(7)$ and $\left(7^{\prime}\right)$ is much too small to be detectable with present experimental techniques.

\section{Electron Capture by $\mathrm{B}^{8}, \mathrm{~N}^{13}$, and $\mathrm{O}^{15}$}

The positron emitters $\left(\mathrm{B}^{8}, \mathrm{~N}^{13}\right.$, and $\left.\mathrm{O}^{15}\right)$ whose neutrino fluxes were calculated by $\operatorname{Sears}^{7}$ can also decay in stellar interiors by capturing a continuum electron. ${ }^{8}$ The ratio of the continuum electron-capture probability to the positron emission probability is ${ }^{10,13}$

$$
\tau_{\beta^{+}} / \tau_{\text {e.c. }}=K / f\left(-Z, W_{0}\right)
$$

where $\tau_{\beta^{+}}$is the terrestrial positron decay lifetime, $\tau_{\text {e.c. }}$ is the stellar electron capture lifetime, $K$ the stellar phase-space factor, $f$ the phase-space function used to analyze allowed terrestrial decays, and $W_{0}$ is the maximum positron energy. The stellar phase-space factor for typical solar conditions is ${ }^{14}$

$$
K \cong 4.79 Z\left(1+X_{\mathrm{H}}\right) T_{6}{ }^{-1 / 2} \rho q^{2}(2 p R / \hbar)^{+2\left(\gamma_{0}-1\right)} .
$$

In Eq. (13), $Z$ is the charge of the initial nucleus; $2\left(\gamma_{0}-1\right) \cong-(\alpha Z)^{2} ; X_{\mathrm{H}}$ is the hydrogen mass fraction; $T_{6}$ is the temperature in units of $10^{+6}{ }^{\circ} \mathrm{K} ; \rho$ is the density in $\mathrm{g}-\mathrm{cm}^{-3} ; q$ is the energy in $m_{e} c^{2}$ units $(0.511$ $\mathrm{MeV})$ of the neutrino emitted in the electron-capture process; $p$ is the average momentum of the captured electron; $R$ is the nuclear radius; $\alpha$ is the fine structure constant; and $\hbar$ is Planck's constant divided by $2 \pi$. The last factor in Eq. (13) represents a small correction due to finite nuclear size. The function $f\left(-Z, W_{0}\right)$ is defined in standard nuclear physics texts. ${ }^{15,16}$

\footnotetext{
${ }^{12}$ J. N. Bahcall and R. A. Wolf, Astrophys. J. 139, 622 (1964). 13 J. N. Bahcall, Astrophys. J. 139, 318 (1964).

14 J. N. Bahcall, Phys. Rev. 128, 1297 (1962).

15 E. J. Konopinski, Ann. Rev. Nucl. Sci. 9, 99 (1959).

${ }^{16}$ M. A. Preston, Physics of the Nucleus (Addison-Wesley Publishing Company, Inc., Reading, Massachusetts, 1962).
} 
TABLE II. Electron capture versus positron emission.

\begin{tabular}{|c|c|c|c|}
\hline $\begin{array}{c}\text { Neutrino } \\
\text { emitter }\end{array}$ & $K$ & $f$ & $\left(\tau_{\beta^{+}} / \tau_{\text {e.c. }}\right)_{\text {eenter of }} \odot$ \\
\hline $\mathrm{B}^{8}$ & $1.1 \times 10^{-1}$ & $5.7 \times 10^{+5}$ & $1.9 \times 10^{-7}$ \\
\hline $\mathrm{N}^{13}$ & $3.3 \times 10^{-3}$ & 7.7 & $4.3 \times 10^{-4}$ \\
\hline $\mathrm{O}^{15}$ & $5.9 \times 10^{-3}$ & 36 & $1.6 \times 10^{-4}$ \\
\hline
\end{tabular}

Note that the neutrinos emitted in the electron capture processes (9)-(11) have $2 m_{e} c^{2}(1.022 \mathrm{MeV})$ more energy than the neutrinos emitted in the corresponding positron decay processes (2)-(4).

In Table II, we present values for $K, f$, and $\left(\tau_{\beta^{+}} / \tau_{\text {e.c. }}\right)$ for processes (9)-(11). The values of $f$ were obtained by direct numerical integration of the defining equa$\operatorname{tion}^{15,16}$ and include finite nuclear size and relativistic corrections. In obtaining Table II, we have used the temperature and density at the center of the sun given by the basic model of Sears. ${ }^{7}$ The solar average of $\left(\tau_{\beta^{+}} / \tau_{\text {e.c. }}\right)$ is approximately equal to $\frac{2}{3}$ the central value listed in Table II.

The neutrino flux for any of the electron-capture processes (9)-(11) is given by the following equation:

$$
\begin{aligned}
\varphi_{\nu}(\text { e.c. }) & =\left(\tau_{\beta^{+}} / \tau_{\text {e.c. }}\right)_{\text {average }} \varphi_{\nu}\left(\beta^{+}\right), \\
& \cong \frac{2}{3}\left(\tau_{\beta^{+}} / \tau_{\text {e.c. }}\right)_{\text {center of } \odot \varphi_{\nu}\left(\beta^{+}\right),}
\end{aligned}
$$

where $\varphi_{\nu}\left(\beta^{+}\right)$is the appropriate positron-emission neutrino flux listed in Table I. It is shown in Sec. IV that the neutrino fluxes from processes (9)-(11), when combined with the appropriate absorption cross sections, are too small to be important in Davis's proposed neutrino experiment.

\section{ABSORPTION CROSS SECTIONS FOR GROUND-STATE TRANSITIONS}

In this section, we give the cross section for the reaction

$$
\nu+(Z, A) \rightarrow e^{-}+(Z+1, A),
$$

and apply this general result to the calculation of absorption cross sections for transitions from the ground state of $\mathrm{Cl}^{37}$ to the ground state of $\mathrm{Ar}^{37}$ induced by solar neutrinos from processes (1)-(11). We assume in this section that the electrons produced by the reaction

$$
\nu_{\text {solar }}+{ }_{17} \mathrm{Cl}^{37} \rightarrow e^{-}+{ }_{18} \mathrm{Ar}^{37}
$$

are all created in continuum states. In Sec. V, the theory of bound-state neutrino absorption is briefly described and the results are applied to the calculation of cross sections for reaction (16).

\section{A. General Formulas}

The theory of nuclear beta decay ${ }^{15,16}$ can be used to calculate the cross section for reaction (15). We find

$$
\sigma_{c}=G_{v}{ }^{2} \xi p_{e} W_{e} F\left(Z, W_{e}\right) / \pi c^{3} \hbar^{4},
$$

where $G_{v}$ is the beta-decay coupling constant; $\xi$ is the usual $^{10,15}$ allowed combination of nuclear matrix elements; $p_{e}$ and $W_{e}$ are the momentum and energy, respectively, of the electron that is produced; and $F\left(Z, W_{e}\right)$ is the familiar Fermi function ${ }^{15}$ which takes account of Coulomb and nuclear size corrections. Equation (17) reduces to the standard ${ }^{17}$ absorption cross section for antineutrinos incident on protons if $F$ is set equal to unity and $\xi$ is set equal to its single-particle value.

We are primarily interested in cross sections for reaction (16). Since $\mathrm{Ar}^{37}$ decays on earth by electron capture, the product $G_{v}{ }^{2} \xi$ that occurs in Eq. (17) can be eliminated, for transitions between ground states, in favor of the experimentally measured $\mathrm{Ar}^{37}$ electron capture lifetime and $L / K$ capture ratio. We obtain

where

$$
\sigma_{c}=\sigma_{0} w_{e}^{2} G\left(Z, w_{e}\right)
$$

$$
\sigma_{0} \equiv \frac{2 \pi \alpha Z \ln 2\left(m_{e} c^{2} / q_{1 s}\right)^{2}}{\left(c \tau_{1 / 2}\right)_{\text {e.c. }[1+L / K]\left|\psi_{1 s}(0)\right|^{2}}}
$$

and

$$
w_{e} \equiv W_{e} / m_{e} c^{2} \text {, }
$$

$$
G\left(Z, w_{e}\right) \equiv\left(c p_{e} F\left(Z, W_{e}\right) / 2 \pi \alpha Z W_{e}\right) .
$$

In Eq. (18b), $q_{1 s}$ is the energy of the neutrino produced in the $K$-capture process; $\left(\tau_{1 / 2}\right)_{\text {e.c. }}$ is the laboratory electron capture half-life; $L / K$ is the electron capture ratio; and $\psi_{1 s}(0)$ is the wave function at the nuclear surface of a $K$ electron in the final atom $\left(\mathrm{Ar}^{37}\right)$.

Equations (18) can be used to calculate the absorption cross sections for the monoenergetic neutrinos that are produced by processes (6)-(11). However, the positron decay processes (1)-(5) produce neutrinos with a continuous spectrum of energies. Hence, for the positron decays (1)-(5), the appropriate cross section is

$$
\sigma_{c}=\sigma_{0}\left(\int_{1}^{w_{e} \max } d w_{e} w_{e}^{2} G\left(Z, w_{e}\right) \lambda\left(q_{\nu}\right)\right) / \int_{0}^{q_{\nu} \max } d q_{\nu} \lambda\left(q_{\nu}\right),
$$

where $\lambda\left(q_{\nu}\right)$ is the known ${ }^{15,16}$ neutrino spectrum of the appropriate positron emitter. The neutrino and electron energies in Eq. (19) are related by the conservation of energy, i.e.,

$$
q_{\nu}=\left(W_{e}-W_{0}\right) / m_{e} c^{2},
$$

where $W_{0}$ is the difference between initial and final nuclear masses.

The final state of $\mathrm{Be}^{8}$ that is predominantly populated by $\mathrm{B}^{8}$ beta decay is a broad state; hence the cross sections given by Eq. (19) must, for $\mathrm{B}^{8}$ decays, be averaged over the profile of the final nuclear state. This particular averaging process changes the predicted neutrino absorption cross section by only a small amount and is not included in this section; numerical results for this effect are summarized in Sec. VI E.

\footnotetext{
${ }^{17}$ F. Reines, Ann. Rev. Nucl. Sci. 10, 1 (1960).
} 
TABLE III. Absorption cross sections for ground-state transitions. ${ }^{\mathrm{a}}$

\begin{tabular}{ccc}
\hline $\begin{array}{c}\text { Neutrino emitting } \\
\text { isotope }\end{array}$ & $\left(\sigma / \sigma_{0}\right)_{\text {line }}$ & $\left(\sigma / \sigma_{0}\right)_{\text {continuum }}$ \\
\hline $\mathrm{H}^{1}$ & 9.0 & 0 \\
$\mathrm{Li}^{4}$ & $2 \times 10^{+3}$ & $7.2 \times 10^{+2}$ \\
$\mathrm{Be}^{7}$ & 1.5 & $\cdots$ \\
$\mathrm{B}^{8}$ & $1.6 \times 10^{+3}$ & $4.0 \times 10^{+2}$ \\
$\mathrm{~N}^{13}$ & $2.6 \times 10^{+1}$ & 1.1 \\
$\mathrm{O}^{15}$ & $4.4 \times 10^{+1}$ & 4.1 \\
\hline
\end{tabular}

a The quantity $\sigma_{0}=1.91 \times 10^{-46} \mathrm{~cm}^{2}$.

\section{B. Numerical Results for $\mathrm{Cl}^{37}$}

The value of $\sigma_{0}$ for reaction (16) can be computed accurately by inserting the following data in Eq. $(18 \mathrm{~b})^{18}$ : $q_{1 s}=814 \mathrm{keV},\left(\tau_{1 / 2}\right)_{\mathrm{e} . \mathrm{c} .}=35.0$ days, $L / K=0.100$, and $\left|\psi_{1 s}(0)\right|^{2}=1.189 \times 10^{+28} \mathrm{~cm}^{-3}$. We find

$$
\sigma_{0}=1.91 \times 10^{-46} \mathrm{~cm}^{2} .
$$

The cross sections for neutrino-induced transitions between the ground states of $\mathrm{Cl}^{37}$ and $\mathrm{Ar}^{37}$ are given in Table III; the cross sections presented correspond to the solar emission processes (1)-(11) [except for process (7)]. The cross section for inducing reaction (16) with solar neutrinos from the $\mathrm{He}^{3}\left(e^{-}, \nu\right) \mathrm{H}^{3}$ decay is essentially zero because the average of neutrinos from reaction (7) is much less than the threshold energy $(814 \mathrm{keV})$ for reaction (16). The cross section for inducing reaction (16) with continuum neutrinos from the proton-proton reaction [Eq. (1)] is also essentially zero, since the endpoint energy of the proton-proton reaction $(0.42$ $\mathrm{MeV})$ is less than the threshold energy $(0.81 \mathrm{MeV})$ for the $\mathrm{Cl}^{37}-\mathrm{Ar}^{37}$ transition.

Exchange and overlap corrections $\mathrm{s}^{19}$ are negligible for the cross sections we have calculated. A 5-keV change in the assumed threshold energy for reaction (16), or in the maximum incident neutrino energy, changes the calculated $\mathrm{N}^{13}$ continuum cross section by at most $4 \%$; all other continuum cross sections in Table III are altered by less than $4 \%$ if the threshold energy or maximum neutrino energy is changed by $5 \mathrm{keV}$.

Multiplying the fluxes given in Sec. III by the appropriate cross sections given in Table III, we see that the only solar neutrinos that are important for Davis's experiment are produced by reactions (2) and (8), i.e., $\mathrm{B}^{8}$ and $\mathrm{Be}^{7}$ neutrinos. ${ }^{20}$

${ }^{18}$ The end-point energy is taken from P. M. Endt and C. Van der Leun, Nucl. Phys. 34, 1 (1962), the half-life from measurements performed at Brookhaven National Laboratory [R. Davis, Jr. (private communication) $]$, the $L / K$ ratio from the summary of R. B. Moler and R. W. Fink, Phys. Rev. 131, 821 (1963), and the value of the $K$ electron's wave function at the nucleus from R. E. Watson and A. J. Freeman, ibid. 123, 521 (1961).

19 J. N. Bahcall, Phys. Rev. 129, 2683 (1963).

20 The possible importance of $\mathrm{B}^{8}$ solar neutrinos was first pointed out by W. A. Fowler, Astrophys. J. 127, 551 (1958) and A. G. W Cameron, Ann. Rev. Nucl. Sci. 8, 299 (1958). The importance of $\mathrm{Be}^{7}$ neutrinos was pointed out by $\mathrm{R}$. Davis, Jr. in a private communication to the author (1963). See also Ref. 6.

\section{NEUTRINO ABSORPTION WITH BOUND ELECTRONS PRODUCED}

The results presented in Sec. IV were derived assuming that all electrons produced by reaction (15) are created in continuum orbits; a similar assumption is also made in all other discussions of neutrino absorption cross sections with which the author is acquainted. However, a certain fraction of the electrons that are produced will be created in bound atomic orbits, resulting in an electrically neutral final atom. ${ }^{21}$ The absorption of a neutrino accompanied by the creation of a bound atomic electron and the transformation of a neutron into a proton is the inverse process corresponding to the familiar electron capture process. The theory for this inverse process, which we shall call "boundstate neutrino absorption," is analogous to the theory of bound-state beta decay. ${ }^{22,23}$

The cross section for allowed bound-state neutrino absorption can easily be derived. We find for the effective capture cross section into a single bound atomic orbit $b$

$$
\sigma_{b}=\pi G_{v}{ }^{2} \xi\left|\psi_{b}(0)\right|{ }^{2} P\left(q_{b}\right) / \hbar c .
$$

In Eq. (21a), $q_{b}$ is the energy of the incident neutrinos that are absorbed, i.e.,

$$
q_{b} \equiv \Delta \epsilon(b)+W_{0}-m_{e} c^{2},
$$

where $\Delta \epsilon(b)$ is the difference between initial and final atomic binding energies. The quantity $P\left(q_{b}\right)$ is defined by

$$
\int \varphi(q) \delta\left(E_{i}-E_{f}\right) d q / \int \varphi(q) d q,
$$

where $\varphi(q)$ is the incident neutrino flux. For positron decays,

$$
P\left(q_{b}\right)=\frac{P_{e^{+}} W_{e^{+}}\left(q_{b}\right)^{2}}{\left(m^{4} c^{5}\right) f\left(-Z, W^{\max }\right)} .
$$

A simple approximate formula for the bound-state absorption cross section is

$$
\sigma_{b} / \sigma_{0}=\left[(\alpha Z)^{2} / 2 n_{b}{ }^{3}\right] P\left(q_{b}\right),
$$

where $n_{b}$ is the principal quantum number for state $b$. We have calculated, using Eqs. (21d) and (22), the bound-state absorption cross sections for neutrinos from processes (2)-(4) incident on $\mathrm{Cl}^{37}$; we find that $\sigma_{b}$ is always small compared to the corresponding continuum absorption cross section, $\sigma_{c}$. The largest value of $\left(\sigma_{b} / \sigma_{c}\right)$ for processes (2)-(4) is the value of 0.04 that obtains

${ }^{21}$ This process was suggested to the author by D. S. Hammer. The mathematical justification for neglecting the small widths of the various states involved is the same as for the analogous problem of the absorption of light. See, for example, W. Heitler, The Quantum Theory of Radiation (Oxford University Press, London, 1957), pp. 163-170.

${ }^{22}$ R. Daudel, M. Jean, and M. Lecoin, J. Phys. Radium 8, 238 (1947)

${ }^{23}$ J. N. Bahcall, Phys. Rev. 124, 495 (1961). 
FIG. 1. Levels of interest in the $A$ equals 37 system. Quantities that are predicted, but not yet measured, are shown in parenthesis. Energies are in $\mathrm{MeV}$.

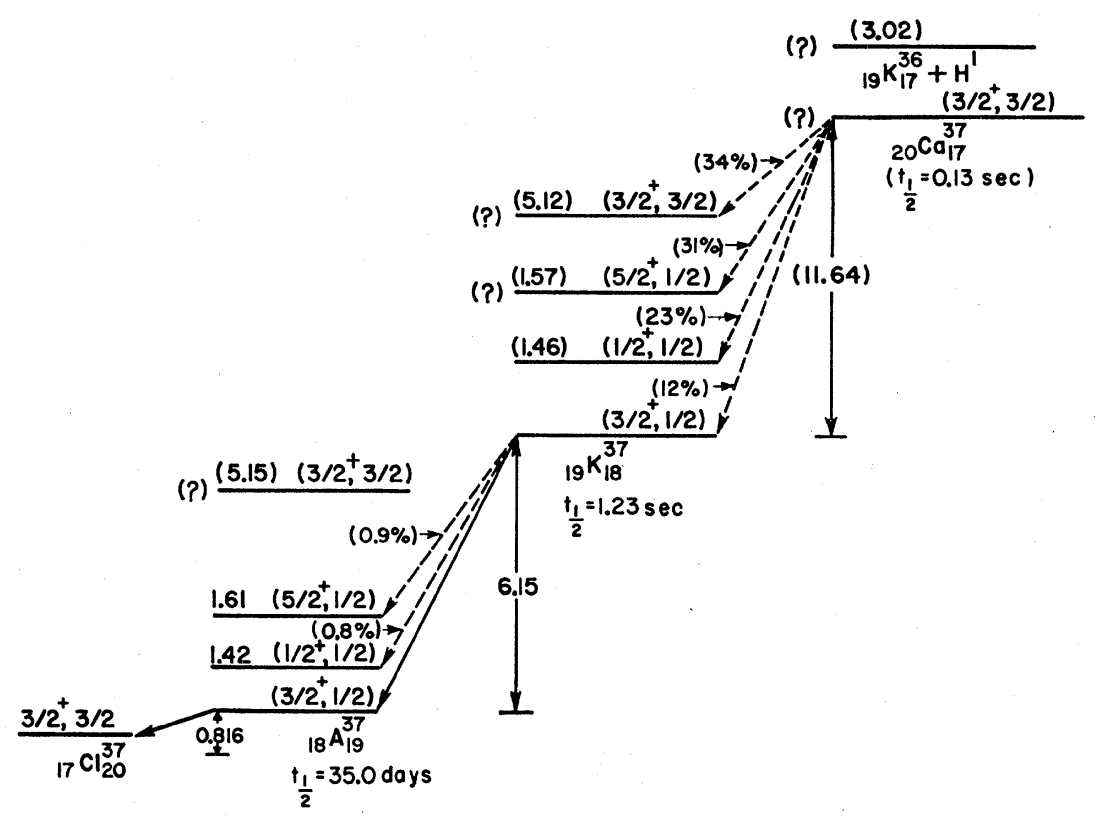

for $\mathrm{N}^{13}$. Hence, bound-state neutrino absorption is not important in Davis's solar neutrino experiment.

Notice, however, that bound-state absorption is a resonance process and $\sigma_{b}$ can become large if $P\left(q_{b}\right)$ is large. Thus, it would be interesting to try to devise an experiment that could exploit the resonance character of bound-state absorption.

\section{TRANSITIONS TO EXCITED STATES}

In this section, we investigate transitions from the ground state of $\mathrm{Cl}^{37}$ to various excited states of $\mathrm{Ar}^{37}$. These transitions, induced primarily by neutrinos from $\mathrm{B}^{8}$ decay, play a dominant role in Davis's experiment. ${ }^{24}$

\section{A. The States and Their Quantum Numbers}

The ground state of ${ }_{17} \mathrm{Cl}_{20}{ }^{37}$ has the quantum numbers (spin, parity, isotopic spin): $J=\frac{3}{2}+, T=\frac{3}{2}$ and a shellmodel configuration (in proton holes) that is $1 d_{3 / 2}$. There are five states in ${ }_{18} \mathrm{Ar}_{19}{ }_{13}$ that can be formed from the $1 d_{3 / 2}^{2}$ proton holes and $1 d_{3 / 2}$ neutron hole; the spins of the five states are, $\frac{1}{2}, \frac{3}{2}$ (two states), $\frac{5}{2}$, and $\frac{7}{2}$, all with positive parity. In the ensuing discussion, we ignore all states in $\mathrm{Ar}^{37}$ that are not primarily composed of three $1 d_{3 / 2}$ holes, since states that are not primarily composed of three $1 d_{3 / 2}$ particles will only give rise to small matrix elements for transitions from the ground state of $\mathrm{Cl}^{37}$. The transition from the $\frac{3}{2}+$ ground state of $\mathrm{Cl}^{37}$ to the $\frac{7}{2}+$ excited state of $\mathrm{Ar}^{37}$ is forbidden and hence the $\frac{7}{2}^{+}$state is also ignored in the following discussion.

The ground state of ${ }_{18} \mathrm{Ar}_{19}{ }^{37}$ presumably has the quantum numbers $J=\frac{3}{2}+, T=\frac{1}{2}$. By analogy with $\mathrm{Cl}^{35}$,

${ }^{24} \mathrm{I}$ am grateful to B. F. Mottelson for a comment that sparked the investigation of excited-state transitions. the two excited states ${ }^{25}$ in $\mathrm{Ar}^{37}$ at 1.4 and $1.6 \mathrm{MeV}$ can very likely be identified with the expected $J=\frac{\mathbf{1}}{\mathbf{2}}{ }^{+}$, $T=\frac{1}{2}$ and $J=\frac{5}{2}+, T=\frac{1}{2}$ states. The $J=\frac{3}{2}+, T=\frac{3}{2}$ state of $\mathrm{Ar}^{37}$ that is the analog of the ground state of $\mathrm{Cl}^{37}$ should have an excitation energy of $5.15 \mathrm{MeV}$; this prediction is obtained by using the approach described by de Shalit and Talmi, ${ }^{26}$ which includes shell and pairing effects.

The relevant levels in $\mathrm{Ar}^{37}$ and $\mathrm{Cl}^{37}$ are shown in Fig. 1, along with some related levels in $\mathrm{K}^{37}$ and $\mathrm{Ca}^{37}$ that will be discussed in Sec. VII. Levels that have not yet been identified experimentally are marked by adjacent question marks. Energy, spin, and isotopic spin values that are predicted, but not yet measured, are shown in parenthesis.

Note that the $B^{8}$ neutrinos from reaction (2) are the only solar neutrinos, according to the discussion of Secs. II and III, that have enough energy and flux to significantly populate excited states of $A r^{37}$.

\section{B. General Formulas}

The absorption cross section for neutrinos to induce an allowed transition between an initial nuclear state $I$ and a final nuclear state $I^{\prime}$ can be shown to be

$$
\sigma=\left(4 \pi^{3} \alpha Z \ln 2 / c\left(f \tau_{1 / 2}\right)_{I ; I^{\prime}}\right)(\hbar / m c)^{3} w_{e}^{2} G\left(Z, w_{e}\right),
$$

where $w_{e}$ and $G\left(Z, w_{e}\right)$ are defined by Eqs. (18c) and (18d), respectively. The quantity $\left(f \tau_{1 / 2}\right)_{I ; I^{\prime}}$ equals the experimental $f t$ value for an observed transition and in

\footnotetext{
${ }^{25}$ P. M. Endt and C. Van der Leun, Ref. 18.

${ }^{26}$ A. de Shalit and I. Talmi, Nuclear Shell Theory (Academic Press Inc., New York, 1963), Chap. 30.
} 
TABLE IV. Transitions among $d_{3 / 2}{ }^{3}$ states.

\begin{tabular}{|c|c|c|c|c|c|c|}
\hline $\begin{array}{l}\text { Initial nuclear state } \\
\qquad\left(J^{\pi}, T\right)\end{array}$ & $\begin{array}{l}\text { Final nuclear state } \\
\qquad\left(J^{\pi}, T\right)\end{array}$ & $\begin{array}{l}\text { Nature of } \\
\text { transition }^{\mathbf{2}}\end{array}$ & $\langle 1\rangle^{2}$ & $\langle\sigma\rangle^{2}$ & $\langle f t\rangle_{\mathrm{Th}}$ & $\langle f t\rangle_{\exp }$ \\
\hline${ }_{17} \mathrm{Cl}^{37}\left(\frac{3}{2}+, \frac{3}{2}\right)$ & ${ }_{18} \operatorname{Ar}^{37}\left(\frac{3}{2}+\frac{3}{2}\right)$ & $\mathrm{sa}$ & 3 & $\frac{1}{5}$ & 0.61 & \\
\hline${ }_{17} \mathrm{Cl}^{37}\left(\frac{3}{2}+, \frac{3}{2}\right)$ & ${ }_{18} \operatorname{Ar}^{37}\left(\frac{3}{2}+, \frac{1}{2}\right)$ & $\mathrm{a}$ & 0 & $4 / 25 x$ & $8.9 \chi$ & 36.8 \\
\hline${ }_{17} \mathrm{Cl}^{37}\left(\frac{3}{2}+, \frac{3}{2}\right)$ & ${ }_{18} \operatorname{Ar}^{37}\left(\frac{1}{2}+, \frac{1}{2}\right)$ & $\mathrm{a}$ & 0 & $3 / 5 \chi$ & 9.8 & \\
\hline${ }_{17} \mathrm{Cl}^{37}\left(\frac{3}{2}+, \frac{3}{2}\right)$ & ${ }_{18} \operatorname{Ar}^{37}\left(\frac{5}{2}+\frac{1}{2}\right)$ & $\mathrm{a}$ & 0 & $21 / 25 x$ & 7.0 & \\
\hline${ }_{19} \mathrm{~K}^{37}\left(\frac{3}{2}+, \frac{1}{2}\right)$ & ${ }_{18} \mathrm{Ar}^{37}\left(\frac{3}{2}+\frac{1}{2}\right)$ & $\mathrm{sa}$ & 1 & $121 / 375$ & $1.37 \pm 0.02$ & $1.42 \pm 0.08$ \\
\hline${ }_{19} \mathrm{~K}^{37}\left(\frac{3}{2}+\frac{1}{2}\right)$ & ${ }_{18} \operatorname{Ar}^{37}\left(\frac{1}{2}+\frac{1}{2}\right)$ & $\mathrm{a}$ & 0 & $4 / 25 x$ & 37 & $?$ \\
\hline${ }_{19} \mathrm{~K}^{37}\left(\frac{3}{2}+, \frac{1}{2}\right)$ & ${ }_{18} \mathrm{Ar}^{37}\left(\frac{5+}{2}, \frac{1}{2}\right)$ & $\mathrm{a}$ & 0 & $28 / 125 x$ & 26 & $?$ \\
\hline${ }_{20} \mathrm{Ca}^{37}\left(\frac{3}{2}+, \frac{3}{2}\right)$ & ${ }_{19} \mathrm{~K}^{37}\left(\frac{3}{2}+, \frac{3}{2}\right)$ & $\mathrm{sa}$ & 3 & $\frac{1}{5}$ & 0.61 & $?$ \\
\hline${ }_{20} \mathrm{Ca}^{37}\left(\frac{3}{2}+, \frac{3}{2}\right)$ & ${ }_{19} \mathrm{~K}^{37}\left(\frac{3}{2}+, \frac{1}{2}\right)$ & $\mathrm{a}$ & 0 & $4 / 25 x$ & 37 & $?$ \\
\hline${ }_{20} \mathrm{Ca}^{37}\left(\frac{3}{2}+, \frac{3}{2}\right)$ & ${ }_{19} \mathrm{~K}^{37}\left(\frac{1}{2}+\frac{1}{2}\right)$ & $\mathrm{a}$ & 0 & $3 / 5 \chi$ & 9.8 & $?$ \\
\hline${ }_{20} \mathrm{Ca}^{37}\left(\frac{3}{2}+, \frac{3}{2}\right)$ & ${ }_{19} \mathrm{~K}^{37}\left(\frac{5}{2}+\frac{1}{2}\right)$ & a & 0 & $21 / 25 x$ & 7.0 & $?$ \\
\hline
\end{tabular}

a sa =superallowed; $\mathrm{a}=$ allowed.

general satisfies the relation

$$
\left(f \tau_{1 / 2}\right)_{I ; I^{\prime}}=\frac{2 \pi^{3} \ln 2\left(\hbar / m_{e} c\right)^{7} m_{e}{ }^{2} c^{3}}{G_{v}{ }^{2} \xi} .
$$

In Eq. (24), $G_{v}$ is the vector coupling constant for nuclear beta decay, ${ }^{27}$

$$
G_{v} \cong(1.41 \pm 0.01) \times 10^{-49} \mathrm{erg}-\mathrm{cm}^{3},
$$

and $\xi$ is the usual combination of reduced matrix elements that obtain for allowed decays,

$$
\xi \equiv\langle 1\rangle^{2}+C_{A^{2}} / C_{V}{ }^{2}\langle\sigma\rangle^{2} .
$$

The experimental value for $C_{A}^{2} / C_{V}^{2}$ is $^{28,29}$

$$
C_{A}{ }^{2} / C_{V}{ }^{2}=1.41 \pm 0.05 \text {. }
$$

For allowed electron capture, one can show that (neglecting $M$ and higher transitions):

$$
f_{\text {e.c. }}=2 \pi^{2}\left(\frac{q_{1 s}}{m_{e} c^{2}}\right)^{2}\left|\psi_{1 s}(0)\right|^{2}\left(1+\frac{L}{K}\right)\left(\frac{\hbar}{m_{e} c}\right)^{3} .
$$

Equation (23) reduces to Eq. (18), for the transition from the ground state of $\mathrm{Cl}^{37}$ to the ground state of $\mathrm{Ar}^{37}$, when one inserts relation (28) in Eq. (23).

Equations (23)-(27) can be used, in conjunction with nuclear-model predictions of $\xi$, to calculate the absorption cross section for a neutrino-induced transition whose inverse reaction is not experimentally observed. For a transition from the ground state of $\mathrm{Cl}^{37}$ to an excited state $I^{\prime}$ of $\mathrm{Ar}^{37}$, it is convenient to use the

${ }^{27}$ R. K. Bardin, C. A. Barnes, W. A. Fowler, and P. A. Seeger, Phys. Rev. 127, 583 (1962).

${ }^{28}$ A. Sosnovskii, P. E. Spivak, Iu. A. Prokof'ev, I. E. Kutikov, and Iu. P. Dobrynin, Zh. Eksperim. i Teor. Fiz. 35, 1059 (1958) [English transl.: Soviet Phys.-JETP 8, 739 (1959)].

${ }_{29}$ E. J. Konopinski, to be published as a chapter in Alpha-Betaand Gamma-Ray Spectroscopy, edited by K. Siegbahn (NorthHolland Publishing Company, Amsterdam, 1964). following relations:

$$
\begin{gathered}
\sigma=\sigma_{0} \frac{\left(f \tau_{1 / 2}\right)_{\mathrm{g} . \mathrm{s} .}}{\left(f \tau_{1 / 2}\right)_{I^{\prime}}} w_{e}^{2} G\left(Z, w_{e}\right), \\
\equiv 36.8 \frac{\sigma_{0}}{\left\langle f \tau_{1 / 2}\right\rangle_{I^{\prime}}} w_{e}^{2} G\left(Z, w_{e}\right) .
\end{gathered}
$$

In obtaining Eq. (29b), we have made use of the fact that for the ground-state transitions discussed in Sec. V

$$
\left(f \tau_{1 / 2}\right)_{\mathrm{g} . \mathrm{s} .}=1.14 \times 10^{+5} \mathrm{sec} .
$$

The reduced $f t$ values, which are convenient for characterizing superallowed and allowed decays, that were introduced in Eq. (29b) are defined by the relations

$$
\begin{aligned}
\left\langle f \tau_{1 / 2}\right\rangle_{I^{\prime}} & =\left(f \tau_{1 / 2}\right)_{I^{\prime}} /\left(f \tau_{1 / 2}\right)_{\mathrm{O}^{14} \rightarrow \mathrm{N}^{14 *}} \\
& =2 C_{V^{2}} /\left(\langle 1\rangle^{2} C_{V^{2}}+\langle\sigma\rangle^{2} C_{A}^{2}\right),
\end{aligned}
$$

where $^{27}$

$$
\left(f \tau_{1 / 2}\right)_{\mathrm{O}^{14} \rightarrow \mathrm{N}^{14}}=3.1 \times 10^{+3} \mathrm{sec} .
$$

\section{Transitions to the $J=\frac{3}{2}+, T=\frac{3}{2}$ State}

The transition from the ground state of $\mathrm{Cl}^{37}$ to the $J=\frac{3}{2}+, T=\frac{3}{2}$ excited state of $\mathrm{Ar}^{37}$ is superallowed, since the states are members of the same isotopic quartet. Hence, for this transition

$$
\langle 1\rangle^{2}=3 .
$$

The results of Freeman et $a l .^{30}$ on $0^{+} \rightarrow 0^{+}$transitions suggest that Eq. (32) should be accurate to better than $5 \%$; the result given in Eq. (32) is also implied by the

${ }^{30}$ J. M. Freeman, J. H. Montague, D. West, and R. E. White, Phys. Letters 3, 136 (1962); J. M. Freeman, J. H. Montague, G. Murray, R. E. White, Phys. Letters (to be published). The results obtained by these authors yield values for $G_{v}{ }^{2}$ for the $\mathrm{Al}^{26 *}, \mathrm{Cl}^{34}$, $\mathrm{V}^{46}$, and $\mathrm{Co}^{54}$ decays that agree to considerably better than $5 \%$ with the value of $G_{v}{ }^{2}$ obtained in Ref. 27 for the $\mathrm{O}^{14}$ decay. 
conserved vector current (CVC) theory. ${ }^{31}$ The GamowTeller matrix element can be calculated by constructing eigenfunctions of total angular momentum and isotopic spin from the basic $1 d_{3 / 2}{ }^{3}$ configuration and, in addition, assuming perfect overlap between the initial and final $1 d_{3 / 2}$ single-particle wave functions. In this way, we find

$$
\langle\sigma\rangle^{2}=\frac{1}{5} \text {. }
$$

These results are summarized in row 1 of Table IV. Note that in Table IV reduced $f t$ values, defined by Eqs. (31), are used.

\section{Transitions to the $T=\frac{1}{2}$ States}

The matrix elements for transitions to the three $T=\frac{1}{2}$ states of $\mathrm{Ar}^{37}$ can be calculated by constructing eigenfunctions in the way discussed in the previous subsection. The imperfect overlap between initial and final single particle states can be estimated by comparing the theoretical $f t$ value with the measured $f t$ value for the $\mathrm{Ar}^{37} \rightarrow \mathrm{Cl}^{37}$ transition between ground states. Let $\chi$ be the parameter that measures the imperfect overlap of the appropriate products of single-particle radial wave functions. Then, for the ground-state transition,

and

$$
\begin{aligned}
& \langle 1\rangle^{2} \equiv 0, \\
& \langle\sigma\rangle^{2}=4 / 25 \chi,
\end{aligned}
$$

$$
\left\langle f \tau_{1 / 2}\right\rangle_{\text {Theoretical }}=8.9 \chi \text {. }
$$

Since the experimental value of $\left\langle f \tau_{1 / 2}\right\rangle$ is 37 , we conclude that $\chi \cong 4$. The reduced matrix elements and $f t$ values for the other transitions of interest in $\mathrm{Ar}^{37}$ are given in rows 3 and 4 of Table IV, assuming that $\chi$ is the same for all transitions.

\section{E. $B^{8}$ Solar Neutrino Cross Sections}

The neutrinos from $\mathrm{B}^{8}$ decay can induce allowed transitions to all four of the $\mathrm{Ar}^{37}$ states discussed in the previous subsections. The cross sections for the various transitions can be calculated from Eq. (29b) and Table IV, once the excitation energies of the various states are known; the cross sections that are calculated must, of course, be averaged over the $\mathrm{B}^{8}$ neutrino spectrum in the manner indicated by Eq. (19). In addition, the $\mathrm{B}^{8}$ cross sections must also be averaged over the profile of the approximately $2 \mathrm{MeV}$ wide $\mathrm{Be}^{8}$ state that is predominantly populated in the $\mathrm{B}^{8}$ beta decay. This has been done using the experimental profile, corrected for beta-decay phase space, obtained by Alburger et al. ${ }^{32}$ The net result is an approximately $5 \%$ decrease in all predicted $\mathrm{B}^{8}$ cross sections compared to the values that were computed assuming a sharp state

${ }^{31}$ R. P. Feynman and M. Gell-Mann, Phys. Rev. 109, 193 (1958); S. S. Gershtein and I. B. Zel'dovich, Zh. Eksperim. i Teor. Fiz. 29, 698 (1955) [English transl.: Soviet Phys.-JETP 2, 576 (1956)].

32 D. Alburger, P. F. Donovan, and D. H. Williams, Phys. Rev. 132, 334 (1963).

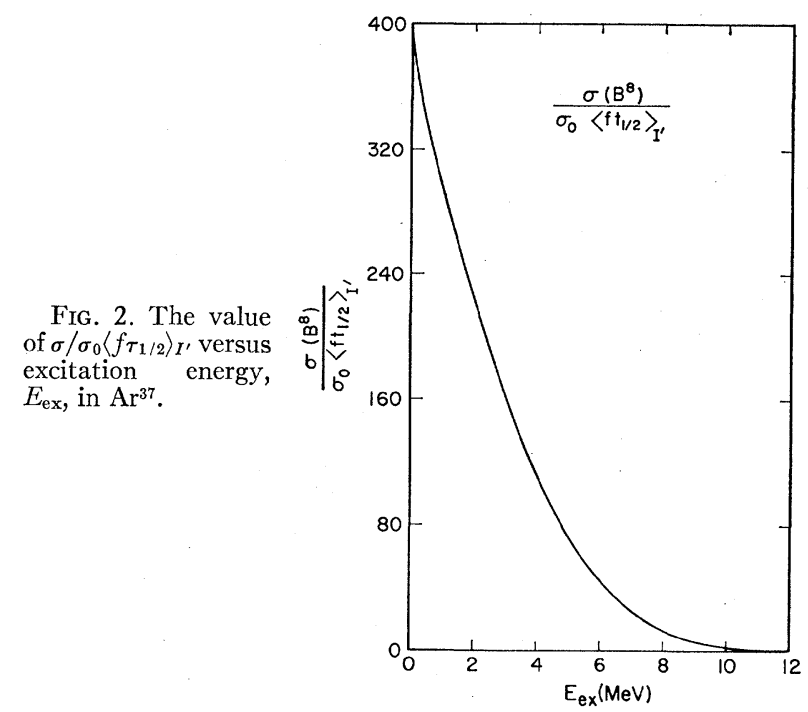

at $2.9-\mathrm{MeV}$ excitation in $\mathrm{Be}^{8}$. This correction is somewhat uncertain due to our, at present, imperfect understanding of the high-energy tail in the alpha-particle spectrum from $\mathrm{Be}^{8 *}$ decay and the possibly related question of what fraction of $\mathrm{B}^{8}$ decays populate the $T=1$ analog state at $16.6-\mathrm{MeV}$ excitation in $\mathrm{Be}^{8}$. In order to illustrate the dependence of the cross section upon the excitation energies of the relevant levels in $\mathrm{Ar}^{37}$, we have plotted in Fig. 2 the value of $\sigma / \sigma_{0}\left\langle f \tau_{1 / 2}\right\rangle_{I^{\prime}}$ versus excitation energy, $E_{\mathrm{ex}}$, in $\mathrm{Ar}^{37}$. Notice that the cross sections depend rather sensitively on the assumed energies for the various excited states. In what follows, we have adopted the values for the energies given in Fig. 1.

The calculated cross sections for transitions to excited states, averaged over the $\mathrm{B}^{8}$ neutrino spectrum and over the profile of the $2.9-\mathrm{MeV}$ "state" in $\mathrm{Be}^{8}$, are (quantum numbers of the excited states in parentheses): $\bar{\sigma}\left(B^{8}\right) / \sigma_{0}=0.99 \times 10^{+3}\left(J=\frac{1}{2}+, T=\frac{1}{2}\right), 1.3 \times 10^{+3}\left(J=\frac{5}{2}+\right.$, $\left.T=\frac{1}{2}\right)$, and $4.1 \times 10^{+3}\left(J=\frac{3}{2}+, T=\frac{3}{2}\right)$. The ground-state cross section, averaged over the neutrino spectrum and the profile of the $2.9-\mathrm{MeV}$ state, is $\bar{\sigma}\left(\mathrm{B}^{8}\right) / \sigma_{0}=0.39 \times 10^{+3}$. Thus, the total absorption cross section for groundstate and excited-state transitions by $\mathrm{B}^{8}$ is

$$
\left[\bar{\sigma}\left(\mathrm{B}^{8}\right) / \sigma_{0}\right]_{\text {total }}=(6.8 \pm 1.5) \times 10^{+3} .
$$

Only about $6 \%$ of the total predicted cross section is due to ground-state transitions.

\section{F. Estimate of Uncertainties}

The estimate of the uncertainty in $\left[\bar{\sigma}\left(\mathrm{B}^{8}\right)\right]_{\text {total }}$ that is given in Eq. (34) was obtained by assuming that the uncertainty in the rate of the superallowed transition discussed in Sec. VI C is about $5 \%$ and the uncertainty in the rate of both of the excited-state transitions discussed in Sec. VI D is about $50 \%$. The small estimated uncertainty in the prediction of the superallowed rate is based 
upon (i) the experimental results of Freeman et $a l .{ }^{30}$ for the Fermi matrix elements of $0^{+} \rightarrow 0^{+}$superallowed transitions; (ii) the fact that the Gamow-Teller contribution is expected to be small $(\sim 7 \%)$; and (iii) the excellent agreement between theory and experiment for the superallowed decay of $\mathrm{K}^{37}$ (see next section). The estimated uncertainty for the two other excited-state transitions of interest is a guess based upon the rough comparisons between theory and experiment made in Sec. VII. Further experimental work of the kind discussed in Sec. VII is necessary before a more accurate estimate of the uncertainty in the excited-state rates can be made.

\section{G. $\mathbf{L i}^{4}$ Cross Sections}

The cross sections for $\mathrm{Li}^{4}$ neutrinos $\left(Q_{\nu}{ }^{\max } \cong 18.8\right.$ $\mathrm{MeV}$ ) to produce transitions to excited states in $\mathrm{Ar}^{37}$ can be calculated in the manner discussed previously for $\mathrm{B}^{8}$ neutrinos. The calculated absorption cross sections are $\left(\mathrm{Ar}^{37}\right.$ excitation energies in parentheses):

$$
\frac{(36.8) \bar{\sigma}\left(\mathrm{Li}^{4}\right)}{\left\langle f_{\tau_{1 / 2}}\right\rangle_{\Gamma^{\prime}} \sigma_{0}}=544(1.4 \mathrm{MeV}), 522(1.6 \mathrm{MeV}) \text {, }
$$

and $217(5.15 \mathrm{MeV})$.

The total absorption cross section for $\mathrm{Li}^{4}$ neutrinos, including both ground and excited-state transitions, is $\sigma / \sigma_{0}=1.9 \times 10^{+4}$. The cross sections given above differ somewhat from those used in previous investigations ${ }^{1,5}$ of the possible importance of $\mathrm{Li}^{4}$ in solar energy generation. Using the cross sections given above, we find (in the notation of Ref. 5) that $F_{p}\left(\mathrm{He}^{3}\right)<0.2 \times 10^{-2}$ and $C_{\mathrm{He}^{3}}\left(\mathrm{He}^{3}, p\right)<0.3 \times 10^{-2}$.

\section{EXPERIMENTAL TESTS FOR $d_{3 / 2}$ CONFIGURATIONS}

In order to test the accuracy of the nuclear model adopted in Sec. VI, we predict in this section the decay rates for some other transition among nuclei described by $d_{3 / 2}$ configurations. We also illustrate a method for analyzing allowed beta decays that, assuming the CVC theory and the constancy of the ratio $C_{A} / C_{V}$, permits one to extract experimental Gamow-Teller matrix elements from $f t$ values for mixed transitions. The experimental energies and lifetimes used in our analysis are taken from the compilation of Endt and Van der Leun. ${ }^{25}$

\section{A. $d_{3 / 2}{ }^{3}$ Transitions $(A=37)$}

The relevant levels in the mass 37 system are shown in Fig. 1, where levels that have not yet been identified experimentally are marked by adjacent question marks and the quantum numbers and energy differences that have not yet been measured are enclosed in parenthesis. The energy differences between the four members of the $J=\frac{3}{2}+, T=\frac{3}{2}$ isotopic quartet were calculated by the method of de Shalit and Talmi. ${ }^{26}$ The energies of the expected $\frac{1}{2}^{+}$and $\frac{5}{2}^{+}$states in $\mathrm{Ar}^{37}$ and $\mathrm{K}^{37}$ were obtained by comparison with the known energies ${ }^{25}$ of these two levels in $\mathrm{Cl}^{35}$ and the approximate location $^{25}$ of two levels in $\mathrm{Ar}^{37}$.

The $\mathrm{K}^{37} \rightarrow \mathrm{Ar}^{37}$ positron decay involves primarily one superallowed decay (the decay between ground states) and two allowed transitions (see Fig. 1). The predicted matrix elements and $f t$ values for these transitions are given in rows 5,6 , and 7 of Table IV. Note that the theoretical reduced $f t$ value for the superallowed decay between the ground states is in excellent agreement with experiment (the small uncertainty in the theoretical prediction is due to the uncertainty in the value of $\left.C_{A} / C_{V}\right)$. The branching to the two excited states is predicted, on the basis of the assumptions outlined in Sec. VI, to be $0.83 \%$ and $0.92 \%$, respectively, as shown in Fig. 1. The $f$ values used in these predictions are given in Table $\mathrm{V}$; they include nuclear size and relativistic effects in the manner described by Fano. ${ }^{33}$ The branching in the $\mathrm{K}^{37}$ decay has not yet been observed experimentally, and, in fact, Endt and Van der Leun ${ }^{25}$ list the decay as going $100 \%$ to the ground state of $\mathrm{Ar}^{37}$. It would be very useful to measure the branching ratios in the $\mathrm{K}^{37} \rightarrow \mathrm{Ar}^{37}$ decays to an accuracy sufficient to test significantly the above predictions.

The isotope ${ }_{20} \mathrm{Ca}^{37}$ is predicted to be particle stable by $3 \mathrm{MeV}$; it has not yet been observed. It would be interesting and useful to measure the total lifetime and branching ratios in the positron decay of $\mathrm{Ca}^{37}$ since ${ }^{34}$ : (i) no superallowed decay among members of the same isotopic quartet $\left(T=\frac{3}{2} \rightarrow T=\frac{3}{2}\right)$ has yet been observed (to the author's knowledge); and (ii) the matrix elements for these decays are identical, except for small Coulomb corrections, with the matrix elements occurring in the $\mathrm{Cl}^{37} \rightarrow \mathrm{Ar}^{37}$ transitions. The reduced matrix elements and $f t$ values for the predicted $\mathrm{Ca}^{37} \rightarrow \mathrm{K}^{37}$ decays are given in rows 8-11 of Table IV; the relevant $f$ values are given in Table $V$. The predicted branching

TABLE V. Some calculated $f$ values.

\begin{tabular}{cccc}
\hline \hline Initial nucleus & Final nucleus & $W_{0}(\mathrm{MeV})$ & $f$ \\
\hline${ }_{16} \mathrm{~S}^{35}$ & ${ }_{17} \mathrm{Cl}^{35}$ & 0.678 & $1.37 \times 10^{-2}$ \\
${ }_{17} \mathrm{Cl}^{33}$ & ${ }_{16} \mathrm{~S}^{33}$ & 5.07 & $2.16 \times 10^{+3}$ \\
${ }_{18} \mathrm{Ar}^{34}$ & ${ }_{17} \mathrm{Cl}^{34}$ & $5.344^{3}$ & $2.76 \times 10^{+3}$ \\
${ }_{18} \mathrm{Ar}^{35}$ & ${ }_{17} \mathrm{Cl}^{35}$ & 5.46 & $3.09 \times 10^{+3}$ \\
${ }_{18} \mathrm{Ar}^{35}$ & ${ }_{17} \mathrm{Cll}^{35}$ & 4.24 & $8.45 \times 10^{+2}$ \\
${ }_{18} \mathrm{Ar}^{35}$ & ${ }_{17} \mathrm{Cl}^{35}$ & 3.70 & $4.17 \times 10^{+2}$ \\
${ }_{19} \mathrm{~K}^{37}$ & ${ }_{18} \mathrm{Ar}^{37}$ & 5.64 & $3.58 \times 10^{+3}$ \\
${ }_{19} \mathrm{~K}^{37}$ & ${ }_{18} \mathrm{Ar}^{37}$ & 4.22 & $8.09 \times 10^{+2}$ \\
${ }_{19} \mathrm{~K}^{37}$ & ${ }_{18} \mathrm{Ar}^{37}$ & 4.03 & $6.37 \times 10^{+2}$ \\
${ }_{20} \mathrm{Ca}^{37}$ & ${ }_{17} \mathrm{~K}^{37}$ & 11.13 & $1.08 \times 10^{+5}$ \\
${ }_{20} \mathrm{Ca}^{37}$ & ${ }_{19} \mathrm{~K}^{37}$ & 9.67 & $5.35 \times 10^{+4}$ \\
${ }_{20} \mathrm{Ca}^{37}$ & ${ }_{19} \mathrm{~K}^{37}$ & 9.56 & $5.05 \times 10^{+4}$ \\
${ }_{20} \mathrm{Ca}^{37}$ & ${ }_{19} \mathrm{~K}^{37}$ & 6.01 & $4.86 \times 10^{+3}$ \\
${ }_{20} \mathrm{Ca}^{39}$ & ${ }_{19} \mathrm{~K}^{39}$ & 5.98 & $4.72 \times 10^{+3}$ \\
\hline
\end{tabular}

a Reference 35 .

${ }^{33}$ U. Fano, Tables for the Analysis of Beta Spectra (Natl. Bur. Std. Appl. Math. Ser. 13, 1952).

${ }_{34} \mathrm{I}$ am grateful to C. A. Barnes, G. Scharff-Goldhaber, M. Goldhaber, and P. A. Seeger for valuable discussions concerning $\mathrm{Ca}^{37}$ and its possible detection. 
TABLE VI. Some other transitions among $d_{3 / 2}$ nuclei.

\begin{tabular}{|c|c|c|c|c|c|c|c|}
\hline $\begin{array}{l}\text { Initial nuclear state } \\
\qquad\left(J^{\pi}, T\right)\end{array}$ & $\begin{array}{c}\text { Final nuclear state } \\
\left(J^{\pi}, T\right)\end{array}$ & $\begin{array}{l}\text { Nature of } \\
\text { transition }^{\mathrm{a}}\end{array}$ & $\langle 1\rangle^{2}$ & $\langle\sigma\rangle^{2}$ & $\langle f t\rangle_{\mathbf{T h}}$ & $\langle f t\rangle_{\exp }$ & $\langle\sigma\rangle_{\exp }{ }^{2}$ \\
\hline $\begin{array}{l}{ }_{16} \mathrm{~S}^{35}\left(\frac{3}{2}+, \frac{3}{2}\right) \\
{ }_{17} \mathrm{Cl}^{33}\left(\frac{3}{2}+, \frac{1}{2}\right) \\
18 \mathrm{Ar}^{34}\left(0^{+}, 1\right) \\
18 \mathrm{Ar}^{35}\left(\frac{3}{2}+\frac{1}{2}\right) \\
18 \mathrm{Ar}^{35}\left(\frac{3}{2}+\frac{1}{2}\right) \\
18 \mathrm{Ar}^{35}\left(\frac{3}{2}+\frac{1}{2}\right) \\
{ }_{20} \mathrm{Ca}^{39}\left(\frac{3}{2}+, \frac{1}{2}\right)\end{array}$ & $\begin{array}{c}{ }_{17} \mathrm{Cl}^{35}\left(\frac{3}{2}+, \frac{1}{2}\right) \\
\mathrm{S}^{33}\left(\frac{3}{2}+\frac{1}{2}\right) \\
{ }_{17} \mathrm{Cl}^{34}\left(0^{+}, 1\right) \\
{ }_{17} \mathrm{Cl}^{35}\left(\frac{3}{2}+, \frac{1}{2}\right) \\
{ }_{17} \mathrm{Cl}^{35}\left(\frac{1}{2}+, \frac{1}{2}\right) \\
{ }_{17} \mathrm{Cl}^{35}\left(\frac{5}{2}+\frac{1}{2}\right) \\
{ }_{19} \mathrm{~K}^{39}\left(\frac{3}{2}+\frac{1}{2}\right)\end{array}$ & $\begin{array}{c}\mathrm{a} \\
\mathrm{sa} \\
\mathrm{sa} \\
\mathrm{sa} \\
\mathrm{a} \\
\mathrm{a} \\
\mathrm{sa}\end{array}$ & $\begin{array}{l}0 \\
1 \\
2 \\
1 \\
0 \\
0 \\
1\end{array}$ & $\begin{array}{c}4 / 25 x \\
\frac{3}{5} \\
0 \\
121 / 375 \\
4 / 25 x \\
28 / 125 x \\
\frac{3}{5}\end{array}$ & $\begin{array}{c}37 \\
1.08 \pm 0.02 \\
1.00 \\
1.37 \pm 0.02 \\
37 \\
26 \\
1.08 \pm 0.02\end{array}$ & $\begin{array}{c}33 \\
1.76 \pm 0.10 \\
? \\
1.79 \pm 0.06 \\
10_{-3}^{+6} \\
12_{-4}^{+12} \\
1.34 \pm 0.04\end{array}$ & $\begin{array}{c}0.043 \\
0.10 \pm 0.05 \\
\ldots \\
0.08 \pm 0.03 \\
0.14 \pm 0.05 \\
0.12 \pm 0.06 \\
0.35 \pm 0.03\end{array}$ \\
\hline
\end{tabular}

a $\mathrm{a}=$ allowed; $\mathrm{sa}=$ superallowed.

ratios are, in order of increasing excitation energy, $12 \%$, $23 \%, 31 \%$, and $34 \%$. The total lifetime of $\mathrm{Ca}^{37}$, including all four branches, is predicted to be $0.13 \mathrm{sec}$.

\section{B. Other $d_{3 / 2}$ Transitions}

The nuclear mode described in Sec. VI can be used to predict transition probabilities for decays among nuclei that do not have $A$ equal to 37 but whose shellmodel configurations are $d_{3 / 2}$ or $d_{3 / 2}^{2}$. Some predictions are shown in Table VI and are compared with experiment wherever possible; the relevant $f$ values are given in Table V. It would be useful to have more accurate data for the decays shown so that more meaningful comparisons with theory could be made. The rather poor agreement between theory and experiment in the mass 33 and 35 decays may reflect the fact that 16 is not a magic number; thus, the assumption that the relevant states are described by configurations constructed solely from $d_{3 / 2}$ single-particle states is probably inaccurate. The disagreement between theory and experiment for the superallowed $\mathrm{Ar}^{35}$ branch is, however, surprisingly large; the experimental result implies that the Gamow-Teller matrix element for this transition is approximately 0.08 instead of the predicted value of 0.32 . The discrepancy between theory and experiment for the $\mathrm{Ca}^{39}$ superallowed decay, about $20 \%$, is also somewhat surprising.

The half-life for $\mathrm{Ar}^{34}$, recently produced by $\mathrm{R}$. Miller of the Kellogg Radiation Laboratory, is predicted to be $1.1 \mathrm{sec}$ if the preliminary value ${ }^{35}(5.34 \mathrm{MeV})$ for $W_{0}$ is correct.

\section{Extraction of Gamow-Teller Matrix Elements}

Gamow-Teller matrix elements for mixed transitions between analog states can be extracted from measured $f t$ values by assuming: (i) the validity of the $\mathrm{CVC}$ theory, and (ii) the constancy of the ratio $C_{A} / C_{V}$. There is now good evidence $e^{29,36,37}$ for the validity of assumption (i), and assumption (ii) is expected on theoretical grounds to be reasonably good for $A$ greater

\footnotetext{
${ }^{35} \mathrm{R}$. Miller (private communication).

${ }^{36}$ Y. K. Lee, L. W. Mo, and C. S. Wu, Phys. Rev. Letters 10 , 253 (1963)

37 M. E. Nordberg, F. B. Morinigo, and C. A. Barnes, Phys. Rev. 125, 321 (1962).
}

than 10 or $15 .{ }^{16}$ Since the Fermi matrix elements for transitions between analog states are given, according to CVC theory, by isotopic-spin coefficients, we can extract experimental values of $\langle\sigma\rangle^{2}$ from the following relation:

$$
\begin{aligned}
\langle\sigma\rangle_{\exp }^{2} & =\left(\frac{2}{\langle f t\rangle_{\exp }}-\langle 1\rangle_{\mathrm{CVC}}^{2}\right) /\left(C_{A} / C_{V}\right)^{2} \\
& \cong\left(\frac{6.2 \times 10^{+3} \mathrm{sec}}{(f t)_{\exp }}-\langle 1\rangle^{2} \mathrm{CVC}\right) / 1.41 .
\end{aligned}
$$

Some experimental values of $\langle\sigma\rangle^{2}$ that were obtained with the help of Eq. (35b) are shown in the last column of Table VI. Note that the accurately measured $\langle\sigma\rangle^{2}{ }_{\exp }$ for the $\mathrm{S}^{35}$ decay is in good agreement with the theoretical prediction; the values of $\langle\sigma\rangle^{2}$ exp for the $\mathrm{Ar}^{35}$ excited-state decays are much less well known and appear to differ significantly from the predicted values. It would be useful to obtain more accurate experimental data for the $\mathrm{Ar}^{35}$ decays.

\section{SUMMARY}

The total predicted number of neutrino captures is

$$
\Sigma \varphi \sigma=(3.6 \pm 2) \times 10^{35} \text { per } \mathrm{Cl}^{37} \text { atom per sec; }
$$

about $10 \%$ of the predicted number of absorptions is due to $\mathrm{Be}^{7}$ neutrinos and about $90 \%$ is due to $\mathrm{B}^{8}$ neutrinos. Uncertainties in the theoretical neutrino absorption cross sections can be reduced, and the interpretation of Davis's proposed solar neutrino experiment simplified, by performing accurately some of the betadecay experiments discussed in Sec. VII.

\section{ACKNOWLEDGMENTS}

I am grateful to Raymond Davis, Jr., and R. L. Sears for many valuable suggestions. It is a pleasure to acknowledge stimulating discussions with C. A. Barnes, J. Eichler, W. A. Fowler, I. Iben, Jr., R. W. Kavanagh, B. R. Mottelson, and M. A. Preston. Many of the calculations were performed with the generous programming assistance of P. A. Seeger and B. A. Zimmerman. 


\section{APPENDIX}

Davis $^{3}$ has shown that the neutral particle $\bar{\nu}_{\beta}$ that appears in negatron emission is different from the neutral particle $\nu_{\beta}$ that appears in positron emission. This difference was established, using antineutrinos from a reactor, by demonstrating that the cross section for $\mathrm{Cl}^{37}\left(\bar{\nu}_{\beta}, e^{-}\right) \mathrm{Ar}^{37}$ was much less than would be expected, on the basis of the measured $\mathrm{Ar}^{37}$ electroncapture lifetime, if $\bar{\nu}_{\beta}$ were equal to $\nu_{\beta}$.

Previous estimates of the expected cross sections for $\mathrm{Cl}^{37}\left(\bar{\nu}_{\beta}, e^{-}\right) \mathrm{Ar}^{37}$ have not included the possibility of excited-state transitions; such transitions may be included using formulas (29) and Table IV of the present work. For use in this connection, we present in Table
TABLE VII. Values of $w_{e}^{2} G\left(18, w_{e}\right)$.

\begin{tabular}{cccc}
\hline$Q_{\nu}(\mathrm{MeV})^{\mathbf{a}}$ & $w_{e}^{2} G\left(18, w_{e}\right)$ & $Q_{\nu}(\mathrm{MeV})^{\mathrm{a}}$ & $w_{e}^{2} G\left(18, w_{e}\right)$ \\
\hline 0.814 & 0.0 & 3.000 & 52.8 \\
0.850 & 1.4 & 4.000 & 99.2 \\
0.875 & 1.7 & 5.000 & 160 \\
0.900 & 1.9 & 6.000 & 235 \\
0.950 & 2.4 & 7.000 & 324 \\
1.000 & 2.9 & 8.000 & 426 \\
1.500 & 10.3 & 10.000 & 675 \\
2.000 & 20.7 & 12.000 & 1004 \\
2.500 & 35.8 & 14.000 & 1539 \\
\hline
\end{tabular}

${ }^{\mathrm{a}} O_{\boldsymbol{p}}(\mathrm{MeV})=0.303+W_{e}(\mathrm{MeV})$.

VII values of $w_{e}^{2} G\left(18, w_{e}\right)$ for neutrino energies that are relevant to Davis's reactor experiment.

\title{
"Old" Resonance Model for $3 \pi$ Decays of $K$ and $\eta$
}

\author{
A. N. Mitra and Shubha Ray \\ Department of Physics and Astrophysics, University of Delhi, Delhi, India \\ (Received 6 February 1964; revised manuscript received 4 April 1964)
}

\begin{abstract}
In view of the current experimental interest in the three-pion modes of $K$ and $\eta$ decays, a phenomenological model of a $\pi-\pi$ resonance in $I=0$, proposed several years ago by one of us, is re-examined in the context of the present experimental data. It is found that the model satisfactorily explains all the available features of $\tau$ and $\tau^{\prime}$ decays if the resonance occurs at an energy around $400 \mathrm{MeV}$ with a width $85-90 \mathrm{MeV}$. The corresponding parameters with a somewhat smaller width $(\sim 75-80 \mathrm{MeV})$ also give satisfactory agreement with the experimental results for $\eta$ decay. This model corresponds to a repulsive $I=0 \pi-\pi$ interaction at low energies and is therefore not in disagreement with the earlier dispersion theoretical predictions for $\tau$ decay, using final-state interactions. It also has a strong resemblance to the $\sigma$ model of Brown and Singer.
\end{abstract}

\section{INTRODUCTION}

$T$ $\mathrm{HE}$ old problem of $\tau$ decay has acquired renewed interest in recent times through the observation of $\eta$-decay events and their great similarity with the former. The current theoretical picture has been aptly summarized by Kacser, ${ }^{1}$ namely, there are two broad alternatives (i) the pion-pole model $^{2}$ in which the momentum dependence of the weak-interaction structure makes itself felt through derivative couplings (for $p$-wave resonances) and (ii) the final-state interaction (fsi) model in which the weak interaction has no structure (i.e., has no momentum dependence). The fsi model got into disrepute after the dispersion theoretic calculations of Khuri and Treiman ${ }^{3}$ showed that the calculated energy spectrum of the unlike pion was incompatible with the requirement of a stronger $I=0$ $\pi-\pi$ force than $I=2$, as believed from other evidence (e.g., low-energy $\pi-N$ scattering). However, the fsi

${ }^{1}$ C. Kacser, Phys. Rev. 130, 355 (1963).

${ }^{2}$ M. A. Baqi Bég and P. de Celles, Phys. Rev. Letters 8, 46 (1962). See also G. Barton and S. P. Rosen, Phys. Rev. Letters 8, 414 (1962); Riazuddin and Fayyazuddin, ibid. 7, 464 (1961).

${ }^{3}$ N. Khuri and S. Treiman, Phys. Rev. 119, 1115 (1960). Also, R. Sawyer and K. C. Wali, ibid. 119, 1429 (1960), model seems to have recovered quite a bit of lost ground through the suggestion of a $J=I=0 \pi-\pi$ resonance (called $\sigma$ ) by Brown and Singer ${ }^{4}$ who have shown that such a "particle" can satisfactorily explain the energy spectrum in the $\tau$ and $\eta$ events. The recent experimental verification by Crawford et al..$^{5}$ of the $\pi^{0}$ spectrum in $\eta \rightarrow \pi^{+}+\pi^{-}+\pi^{0}$ in terms of the Brown-Singer hypothesis provides quite impressive support in its favor, though, of course, it does not confirm the existence of $\sigma$.

As the fsi model is now doing rather well (at least for the time being), we would like to draw attention to a phenomenological model proposed in connection with $\tau$ decay by one of us (ANM) several years ago $^{6}$ when the data were rather poor. Subsequently; a refined version of this model $^{7}$ which we shall refer to in this note as "resonance model," was reported by the same author $(\mathrm{ANM})^{8}$ to produce a qualitatively correct

${ }^{4}$ L. Brown and P. Singer, Phys. Rev. Letters 8, 460 (1962).

5 F. S. Crawford, Jr., R. S. Grossman, L. J. Lloyd, L. R. Price, and E. C. Fowler, Phys. Rev. Letters 11, 564 (1963).

6 A. N. Mitra, Nucl. Phys. 6, 404 (1958); referred to as A.

7 A. N. Mitra, Nucl. Phys. 18, $502(1960)$; referred to as B.

8 A. N. Mitra, in Proceedings of the 1960 International Conference on High-Energy Physics at Rochester (Interscience Publishers, Inc., New York, 1960). 